\title{
Wasserbestimmung im Transformatoröl: ein Beitrag zur Erhöhung der Prozesssicherheit
}

\author{
Ute Sasum $^{1}$, Ulrich Guth ${ }^{2}$ \\ ${ }^{1}$ Forschungszentrum Sensorik Greifswald e.V., Brandteichstraße 19, D-17489 Greifswald \\ ${ }^{2}$ Technische Universität Dresden, Fachbereich Chemie und Lebensmittelchemie, D-01062 Dresden
}

\section{Zusammenfassung}

Die Bestimmung von gelöstem Wasser in Isolierölen ist für die Betriebssicherheit des Transformators von entscheidender Bedeutung. Allerdings werden häufig je nach verwendeter Bestimmungsmethode unterschiedliche Werte gemessen, die zu Unsicherheiten bei der Beurteilung des Betriebszustandes von Transformatoren führen. Im Falle der Karl-Fischer-Methode (KF) werden diese Abweichungen Nebenreaktionen mit Abbauprodukten zugeschrieben. Durch die Untersuchungen konnte gezeigt werden, dass die Messergebnisse der KF-Titration auch dann gestört sein können, wenn die auf Alterungsprodukte hinweisenden Ölkennzahlen nicht oder nur sehr gering erhöht sind. Zur Erhöhung der Prozesssicherheit von Leistungstransformatoren wurde ein Online-Verfahren entwickelt, mit dem es möglich ist, in einem Gerät zur Messung der im Öl gelösten Transformatorgase (TGM, Gatron GmbH, Greifswald) mit einem kapazitiven Sensor die Wasserkonzentration in einem Gleichgewichtsgasraum zu messen und daraus die Konzentration im Öl zu berechnen.

\section{Einleitung}

Ein wichtiges Qualitätskriterium für den Einsatz von Isolieröl in elektrischen Hochspannungsgeräten ist sein Wassergehalt. Er gestattet einerseits die Einschätzung der Isolierwirkung des Öls im Hinblick auf die Betriebssicherheit des Transformators, andererseits kann aus der Wasserkonzentration im Öl der Wassergehalt in der Festisolation berechnet und damit die aktuellen Alterungsrate der Zellulose abgeschätzt werden. Die Bestimmung des Wassergehalts im Öl erfolgt im Laboratorium durch Karl-Fischer-Titration. Seit einigen Jahren werden verstärkt kapazitive Sensoren zur Online-Überwachung der Ölfeuchte direkt in Transformatoren eingebaut. Mit diesen Sensoren wird der relative Wassergehalt im Öl bestimmt. Die Umrechnung in die absolute Wasserkonzentration ist nur dann genügend genau, wenn die von Öltyp und Alterungszustand abhängige Sättigungskonzentration des Wassers für das gemessene Öl bekannt ist. Die Ergebnisse der Karl-Fischer-Titration (KF) und der kapazitiven Messungen im Öl weichen in der Praxis häufig voneinander $\mathrm{ab}$, was $\mathrm{zu}$ Unsicherheiten bezüglich des Betriebszustands und einzuleitender Wartungsmaßnahmen führt. Die vorliegende Arbeit soll durch einen Vergleich beider Messmethoden anhand eines neuen Transformatoröls und eines ausgewählten Betriebsöls einen Beitrag zur Genauigkeit der KFTitration leisten. Es soll ebenfalls untersucht werden, ob es möglich ist, Änderungen der Wasserkonzentration im Öl durch Messung in einer mit dem Öl im Gleichgewicht befindlichen Gasphase zeitnah zu erfassen.

\section{Karl-Fischer-Titration von Isolierölen}

Die Karl-Fischer-Titration ist kein einheitliches Verfahren und erfordert oft angepasste Arbeitstechniken und spezifische Reagenzien. Zur Messung der Ölfeuchte können nach IEC 60814 [1] zwei Messmodi, die direkte Titration und die Ausheizmethode, genutzt werden. Ringversuche an Betriebsölproben zwischen sieben Laboratorien weisen erheblich größere Abweichungen der Ergebnisse auf als in IEC 60814 zur Qualitätssicherung (Vergleichsgrenze) gefordert werden [2].

Isolieröle auf Mineralölbasis sind Kohlenwasserstoffgemische mit sehr vielfältigen chemischen Strukturen. Zur Verbesserung bestimmter Eigenschaften können weitere Verbindungen, z.B. als Oxidationsinhibitoren 
oder Metallpassivatoren zugefügt werden. Im Transformatorbetrieb altern Mineralöle durch thermische und elektrische Belastung. In Bild $\mathbf{1}$ ist ein allgemeines Reaktionsschema für die Bildung von Alterungsprodukten aus Kohlenwasserstoffgemischen dargestellt [3]. Außer den Ölalterungsprodukten können sich im Transformatoröl auch gelöste Zelluloseabbauprodukte, Metallionen (hauptsächlich Kupferund Eisenionen) sowie Partikel unterschiedlicher Herkunft und Zusammensetzung befinden. Diese können Nebenreaktionen während der KF-Titration eingehen (Tafel 1) [4] und prinzipiell zu fehlerhaften Ergebnissen führen.

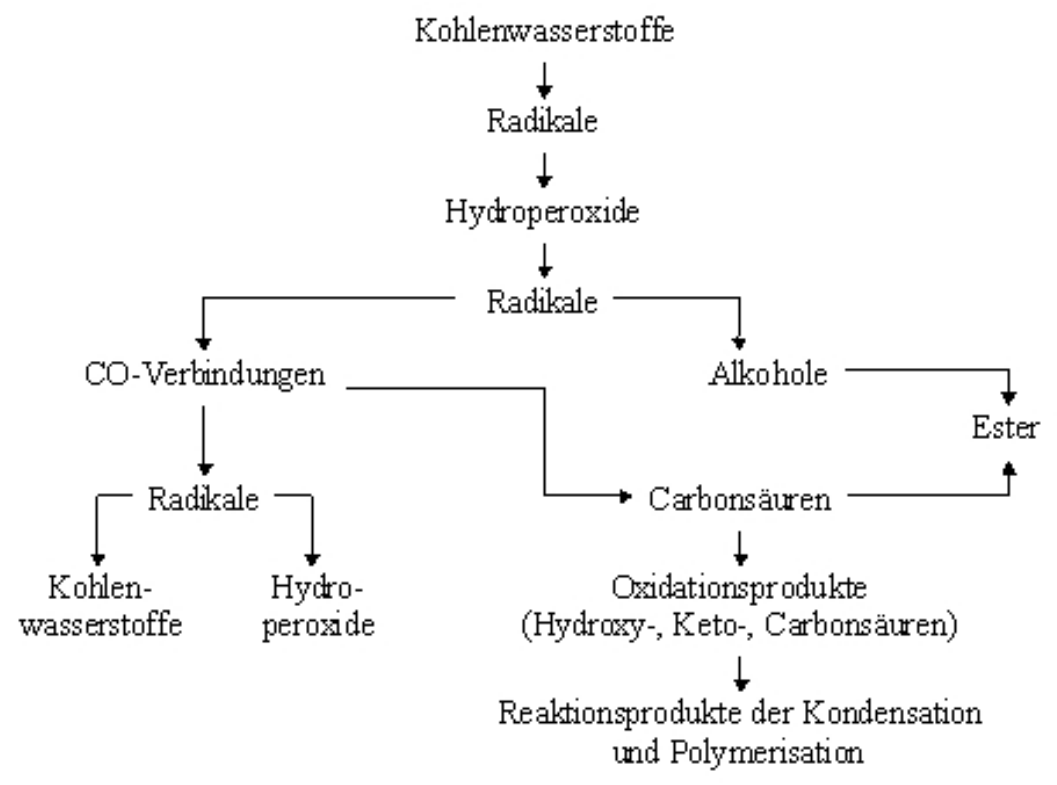

Bild 1 Allgemeines Reaktionsschema für die Bildung von Ölalterungsprodukten

Tafel 1 Mögliche Nebenreaktionen der direkten KF-Titration

\begin{tabular}{l|l|l}
\hline Stoffgruppe & Reaktion & Folge \\
\hline Aldehyde, Ketone & Bildung von Acetalen, Ketalen & Langsame Wasserbildung \\
\hline Kohlenwasserstoffe mit Doppelbindung & $\begin{array}{l}\text { Anlegerung des Iods an die } \\
\text { Doppelbindung }\end{array}$ & Wasser wird vorgetäuscht \\
\hline Mercaptane und Sulfide (Thiole, Thioester) & Qxidation durch Iod zu Disulfiden & Wasser wird vorgetäuscht \\
\hline Starke Reduktionsmittel & Reduzieren Iod & Wasser wird vorgetäuscht \\
\hline Starke Oxidationsmittel (z.B. Peroxide) & Oxidation des Iodids zu Iod & Bestimmung von zu wenig Wasser \\
\hline Metalloxide & Reaktion mit dem KF-Reagenz & Wasserbildung \\
\hline Carbonsäuren & Veresterung & Wasserbildung \\
\hline
\end{tabular}

Durch Anwendung von indirekten Ausheizverfahren können diese Nebenreaktionen teilweise ausgeschlossen oder zumindest reduziert werden. Als Ursache für abweichende Ergebnisse der Wasserbestimmungen von gealterten Transformatorölen, die mit unterschiedlichen Verfahren (photoakustische Spektroskopie, kapazitive Messungen, direkte, indirekte KF-Titration) durchgeführt wurden findet man in der Literatur den Hinweis auf Nebenreaktionen mit Alterungsprodukten, die unter Wasserbildung verlaufen, z.B. Acetal- und Ketalbildung aus Aldehyden und Ketonen [2, 5, 6, 7]. Diese Annahmen wurden aber bisher experimentell nicht belegt.

Die Untersuchungen wurden mit einem coulometrischen Karl-Fischer-Titrator Aqua 40.00 mit/ohne Ölmodul (ECH Elektrochemie Halle $\mathrm{GmbH}$ ) und Apura CombiCoulomat fritless (Merck KGaA) durchgeführt. Bild 2 zeigt die Titrationskurven eines neuen Transformatoröls und eines ausgewählten Betriebsöls. Bei beiden Proben handelt es sich um nicht inhibierte, naphthenische Transformatoröle mit geringem Aromatengehalt. Das Neuöl A erfüllt die Anforderungen der DIN EN 60296 [8]. Die Ergebnisse der Isolieröluntersuchungen des Betriebsöls A (Bild 3) ergeben keine Anhaltspunkte für das Vorhandensein von Alterungsprodukten in größeren Konzentrationen. Trotzdem ist die KF-Titration deutlich gestört. Es werden sowohl bei der Direktinjektion als auch bei den Messungen im Ölmodul bei Raumtemperatur und $120^{\circ} \mathrm{C}$ keine Endwerte in der KF-Titration erreicht. Das trifft auch für eine im Stickstoffstrom getrocknete 
Betriebsölprobe zu. Aus der Differenz der Titrationskurven der nicht getrockneten und der im Stickstoffstrom getrockneten Betriebsölprobe A wurde eine Wasserkonzentration ermittelt, die der im Neuöl A entsprach. Die Wasserkonzentrationen im Neuöl und Betriebsöl wurden unter den gleichen Bedingungen (Gasfeuchte, Temperatur) eingestellt.
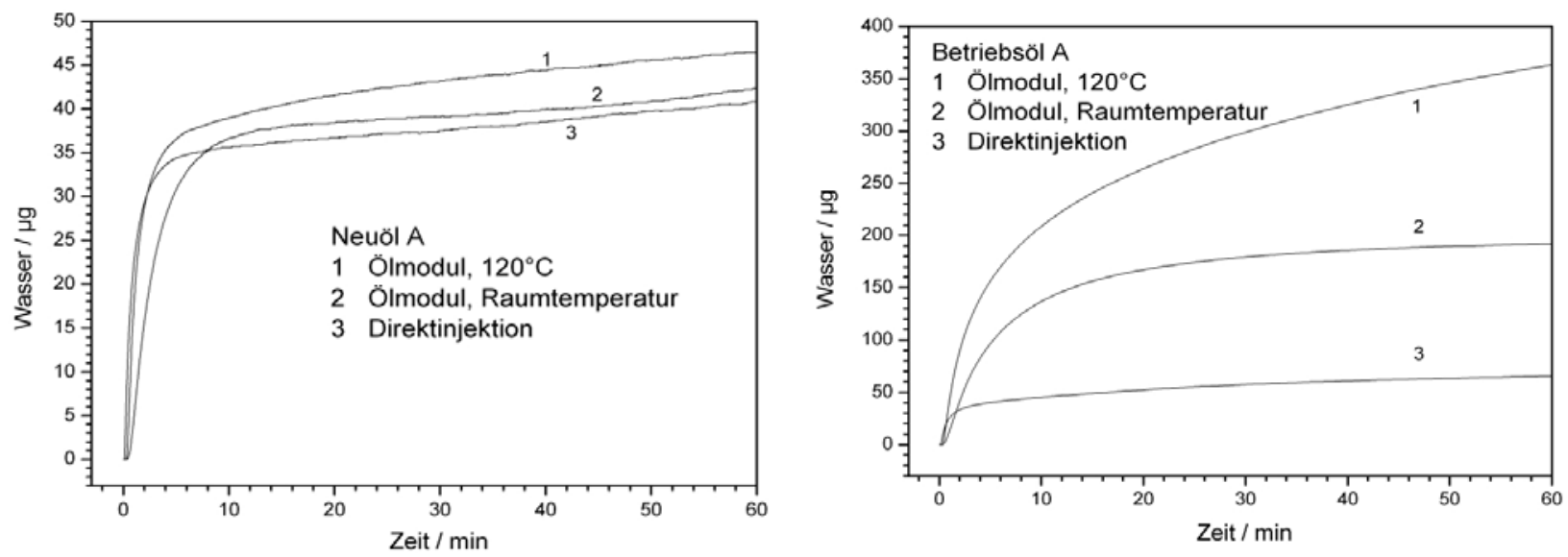

Bild 2 KF-Titration eines neuen Transformatoröls und eines Betriebsöls

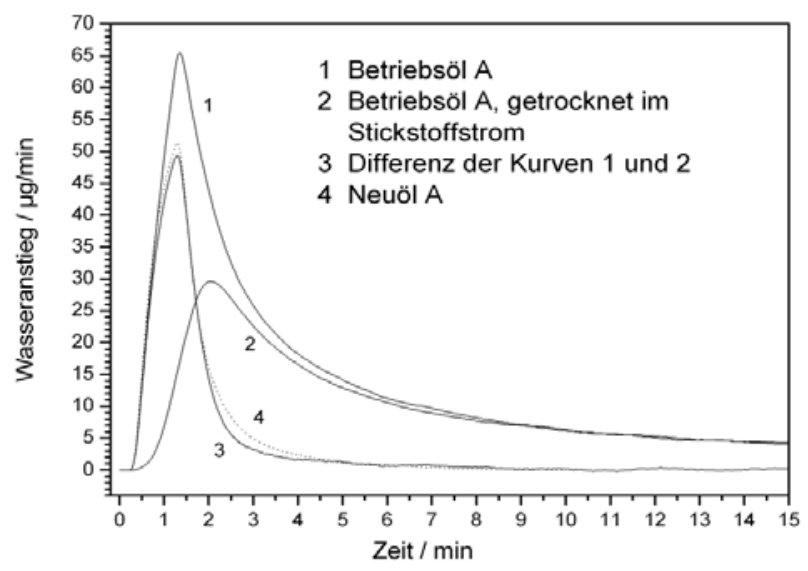

Betriebsöl A:

Durchschlagspannung (DIN EN 156): $75 \mathrm{kV}$

Dielektrischer Verlustfaktor DIN EN 60296): 0,00639

Neutralisationszahl (DIN 51558 T.2): $<0,01 \mathrm{mgKOH} / \mathrm{g}$

Farbzahl (DIN ISO 2049): L1,0

Reinheit DIN 57370 T. 1): klar, keine Teilchen

Gas-in-Öl-Analyse (DIN EN 60567): in ppm

$\begin{array}{lll}\mathrm{H}_{2}: & <10 & \mathrm{CH}_{4}: 1 \\ \mathrm{O}_{2}: & 18063 & \mathrm{C}_{2} \mathrm{H}_{6}: 4 \\ \mathrm{~N}_{2}: & 61114 & \mathrm{C}_{2} \mathrm{H}_{4}: 2 \\ \mathrm{CO}: 60 & \mathrm{C}_{2} \mathrm{H}_{2}:<1 \\ \mathrm{CO}_{2}: 1221 & \mathrm{C}_{3} \mathrm{H}_{8}: 4 \\ & & \mathrm{C}_{3} \mathrm{H}_{6}: 6\end{array}$

Bild 3 KF-Titration im Ölmodul bei $120^{\circ} \mathrm{C}$, Ergebnisse der Isolieröluntersuchung des Betriebsöls A

Im Unterschied zur KF-Titration ergaben Messungen der relativen Ölfeuchte mit einem kapazitiven Sensor im Rahmen der Messgenauigkeit gut übereinstimmende Werte von $52,7 \%$ bei $24,6^{\circ} \mathrm{C}$ für das Neuöl A und $53,0 \%$ bei $24,2^{\circ} \mathrm{C}$ für das Betriebsöl $\mathrm{A}$. Der relative Luftfeuchtegehalt, der aus Messungen mit einem Taupunktspiegelhygrometer ermittelt wurde, betrug $51,7 \%$ bzw. $52,2 \%$.

\section{Bestimmung der relativen Ölfeuchte durch Messung der Gasfeuchte}

Die Verteilung des Wassers in einem Öl-Gas-Gleichgewicht kann in weiten Druck- und Temperaturbereichen mit dem Henry'schen Gesetz beschrieben werden [9]. Im Gleichgewichtszustand sind relative Ölfeuchte und relative Gasfeuchte proportional und in erster Näherung gleich. In Bild 4 sind Messungen der relativen Ölfeuchte (MMT318, Vaisala $\mathrm{GmbH}$ ) und der Gasfeuchte (digitaler kapazitiver Sensor) dargestellt. Beide Sensoren waren in einen Online-Transformatorgas-Monitor TGM (Gatron GmbH) eingebaut. Die Messungen erfolgten an einem Technikumsmessstand mit zwei Ölsystemen unterschiedlicher Feuchte. Durch das Messregime für die im Öl gelösten Gase kann sich das Gleichgewichtsgas im TGM verändern. Zur Ermittlung der Ölfeuchte ist es deshalb wichtig, dass sich das Phasengleichgewicht des Wassers relativ schnell wieder einstellt. Die Bedingungen dafür sind im TGM gegeben. Aus den Kurvenverläufen ist erkennbar, dass die Bestimmung der Ölfeuchte über die Gasfeuchte mit großer Dynamik möglich ist. 


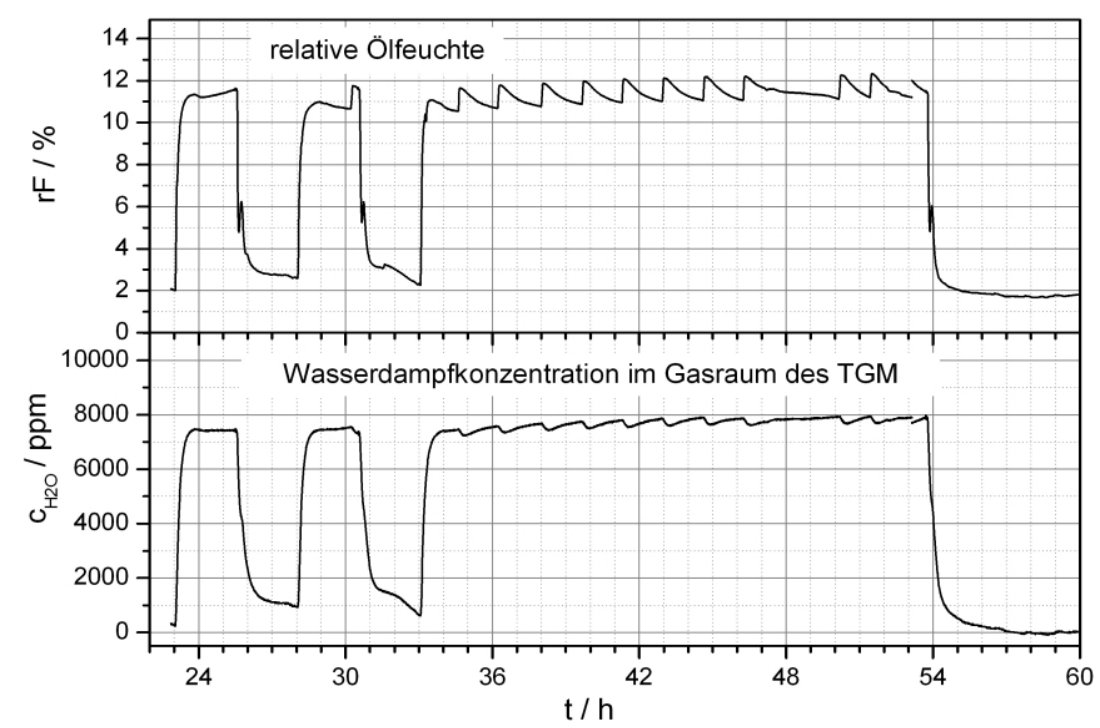

Bild 4 Messung der rel. Ölfeuchte und der Wasserdampfkonzentration im Gleichgewichtsgasraum des TGM

Die Umrechnung der relativen Ölfeuchte in die absolute Wasserkonzentration in $\mathrm{mg} / \mathrm{kg}$ ist nur möglich, wenn die Sättigungskonzentration des jeweiligen Betriebsöls ermittelt wird. Bisher gibt es aber keine gesicherten Erkenntnisse darüber, in welchem Maße und Zeiträumen sich die Sättigungskonzentration durch die Ölalterung im Transformator ändert. Deshalb ist es für die langfristige Online-Überwachung zur Erhöhung der Prozesssicherheit von Leistungstransformatoren vorteilhaft, als Überwachungsgröße die relative Gasfeuchte in einem mit dem Kesselöl des Transformators im Gleichgewicht stehenden Gasraum zu nutzen.

\section{Literatur}

[1] IEC 60814:1997: Isolierflüssigkeiten - Ölimprägniertes Papier und ölimprägnierter Preßspan Bestimmung von Wasser durch automatische coulometrische Karl-Fischer-Titration.

[2] M. Koch, S. Tenbohlen, J. Blennow, I. Höhlein: Reliability and Improvements of Water Titration by the Karl Fischer Technique, 15. ISH, 27.-31.08.2007, Ljubljana, Slovenia.

[3] G. Jäger: Schmierstoffe und ihre Prüfung im Labor, 1. Aufl. Leipzig: VEB Deutscher Verlag für Grundstoffindustrie 1984.

[4] M. Hahn: Wasserbestimmung in Isolierölen, 3. Regensburger Transformatorsymposium, Regensburg 06.- 08.11.2007.

[5] M. Koch: Improved Determination of Moisture in Oil-Paper-Insulations by Specialised Moisture Equilibrium Charts, Proceedings of the XIVth International Symposium on High Voltage Engineering, S. 508, Beijing, China, 2005.

[6] DOMINO Application Note 22: Comparison between the Karl Fischer Titration for Water and the DOMINO Measurement System, Doble Engineering Company, Rev A, 2003.

[7] J. Altmann, Bukvis: The Oil-Moisture Diagnostic Problem of Aged Transformers, 2004, www.ars-altmann.com/News.

[8] DIN IEC 60296:2009: Flüssigkeiten für elektrotechnische Anwendungen - Neue Isolieröle auf Mineralölbasis für Transformatoren und Schaltgeräte.

[9] M. Loncin: Die Löslichkeit des Wassers in Fetten und Ölen und der Dampfdruck des gelösten Wassers, Fette, Seifen, Anstrichmittel, 57 (1955) S. 413-413, zitiert in: K-H- Holle: Über die elektrischen Eigenschaften von Isolierölen, insbesondere über den Einfluß von Wasser auf deren Temperaturverhalten, Dissertation, TU Braunschweig, 1967.

Die Untersuchungen wurden im Rahmen der Verbundforschung in Mecklenburg-Vorpommern durch die Europäische Union, ESF und EFRE gefördert. 